\title{
Generation and Quenching of Intensity Pulsations in Semiconductor Lasers Coupled to External Cavities
}

\author{
KAM Y. LAU, LUIS FIGUEROA, AND AMNON YARIV, FELLOW, IEEE
}

\begin{abstract}
The behavior of self-pulsing and nonpulsing lasers coupled to external cavities is investigated experimentally and theoretically. We investigate the dependence of the pulsation characteristics on the external cavity length using a saturable absorber model for self-pulsing lasers. It was found that quenching of self-pulsation occurs only for a certain limited range of external cavity length, and the frequencies of external-cavity induced pulsations lies within a certain range determined by the coupling coefficient. Small-signal analysis allows these ranges to be derived analytically. Hitherto, complex pulsation phenomena can be explained very intuitively by interpreting the combined laserexternal cavity system as a microwave oscillator with a limited gain band and discrete mode structure.
\end{abstract}

\section{INTRODUCTION}

$\mathrm{T}$ HE behavior of a laser diode coupled to an external cavity has been of considerable interest for many years [1]. Previous studies have focused on the spectral and dynamic behavior of lasers operating in external cavities. Recent studies on the dynamic behavior were particularly interesting, with self-pulsations being quenched [2], [3] , and picosecond pulses [4], [5] being generated. Kuwahara has studied the interaction of self-pulsing lasers coupled to external cavities using a saturable absorber model [6]. It was shown, by numerical calculation, that this model can predict the observed quenching and frequency locking effect. How the quenching effect depends on the external cavity length was not shown. Experimental studies have shown that the length of the external cavity plays a major role in the pulsation characteristics. It was observed that [2], [3] quenching of self-pulsation occurs only over a certain range of external cavity length. Beyond that range, the pulsation frequency locks to an external cavity harmonic [7]. It was found that [3] a trap model can explain this kind of length dependence. When a nonpulsing laser is coupled to an external cavity, pulsations can be induced, with pulses sometimes as short as $5 \mathrm{ps}$ [8]. The induced pulsation frequency also locks to an external cavity harmonic. We have performed similar experiments and found that pulses can be induced only over certain ranges of external cavity

Manuscript received December 4, 1979; revised July 24, 1980. This work was supported by the National Science Foundation under Grant ENG-7604927-A04, the Office of Naval Research under Contract N00014-76-0322, and by DARPA, and monitored by NRL under Contract N00173-78-C-0192.

K. Y. Lau and A. Yariv are with the California Institute of Technology, Pasadena, CA 90265.

L. Figueroa is with the Hughes Research Laboratories, Malibu, CA 90265 .

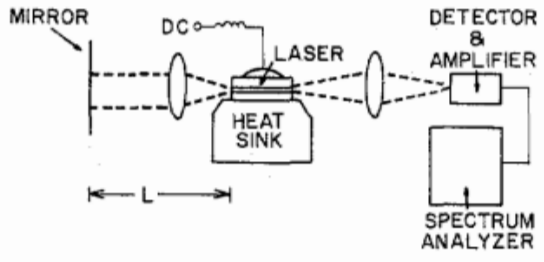

Fig. 1. Experimental setup for coupling of laser diodes to an external cavity.

lengths. This was also predicted by the same trap model [3] used before for the quenching effect, where we have assumed that laser diodes, even though they do not self-pulse, still contain a certain amount of saturable absorber. One has to resort to numerical calculations when dealing with the trap model due to its mathematical complexity, and consequently, lose sight of the role each parameter (coupling coefficient, cavity length, etc.) plays in determinating the solution. Kuwahara's approach [6], however, takes on a much simpler form but is basically similar to the trap model, namely, the saturable absorber effect. (The trap model is actually a combination of saturable absorption plus superlinear gain, but these two effects are indistinguishable in their consequences.) Thus, proceeding along this line and through a small-signal analysis, we derive analytical expressions for the range of external cavity length where quenching and induced pulsing occur. Intuitive understanding in the general cavity-length dependence can be gained by casting the model in the form of a microwave oscillator with a finite gain band, the width of which depends on the coupling coefficient between the laser diode and the external cavity.

\section{EXPERIMENTAL OBSERVATIONS}

Experiments were performed to study the effect of external cavities on various lasers. To make it easy for varying the external cavity length, the output from the lasers is collimated by a $10 \mathrm{X}$ microscope objective, and reflected back by a plane mirror mounted on a translation stage (see Fig. 1). Good coupling is indicated by a lowering of the lasing threshold. After good alignment is obtained, the laser output, detected by an APD with 150 ps rise time, is fed into a microwave spectrum analyzer, and its spectrum is compared to the one without the external cavity. Our observations can be grouped into two categories. 


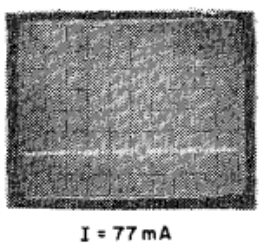

(a)

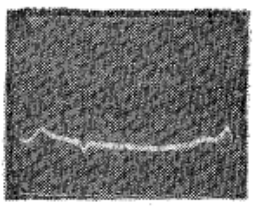

$I=77 \mathrm{~mA}$

$\epsilon=15 \%$

$\mathrm{L}=8.5 \mathrm{~cm}$

(b)

Fig. 2. Microwave spectrum of output of a nonpulsing laser: (a) without external cavity; (b) with external cavity. Horizontal scale: $180 \mathrm{MHz}$ / div. Vertical scale: $10 \mathrm{~dB} /$ div. The notch is a frequency indicator at $540 \mathrm{MHz}$.
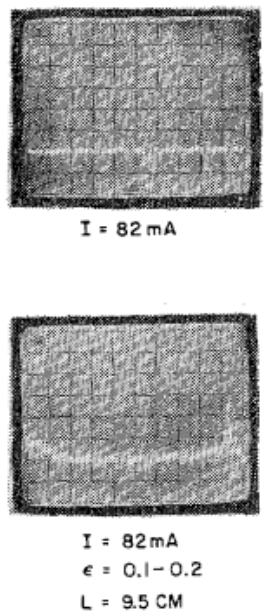

(b)

Fig. 3. Microwave spectrum of output of another nonpulsing laser: (a) without cavity; (b) with external cavity. The notch is a frequency indicator at $1 \mathrm{GHz}$.

\section{A. Nonpulsing Lasers}

We have examined a laser which is relatively new (stripe geometry laser with threshold of about $80 \mathrm{~mA}$ ) and displays a flat microwave spectrum [see Fig. 2(a)], which indicates that no pulsing occurs. No sharp resonance can be induced in the microwave spectrum by aligning it in an external cavity. The coupling coefficient $\epsilon$ (defined as the fraction of light fed back into the laser diode by the external cavity) is estimated from the lowering of lasing threshold to be about 15 percent. However, a broad and weak resonance can be seen [see Fig. 2(b)] peaking at approximately the roundtrip cavity frequency and is more prominent at higher current levels.

We have also examined another stripe geometry laser (threshold $\simeq 80 \mathrm{~mA}$ ) which displays no self-pulsing [see Fig. 3(a)] . When aligned with the external resonator, a dramatic sharp resonance occurs at the fundamental external cavity roundtrip frequency of $1.6 \mathrm{GHz}$. The peak of the resonance is almost $30 \mathrm{~dB}$ above background level [see Fig. 3(b)], with

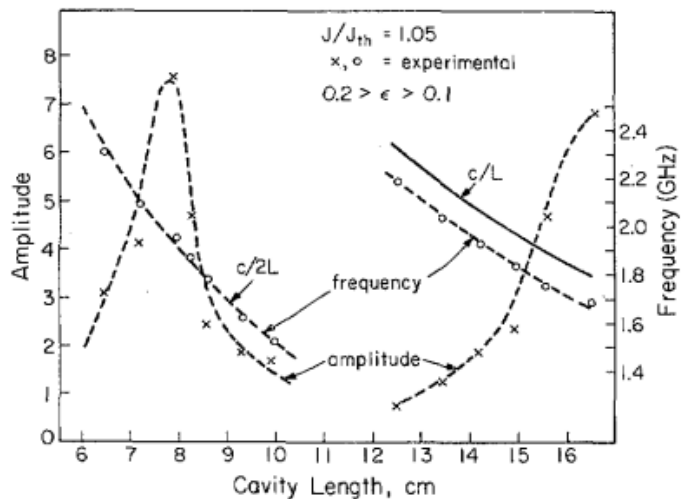

Fig. 4. Experimental plot of amplitude and frequency of induced resonance versus external cavity length.

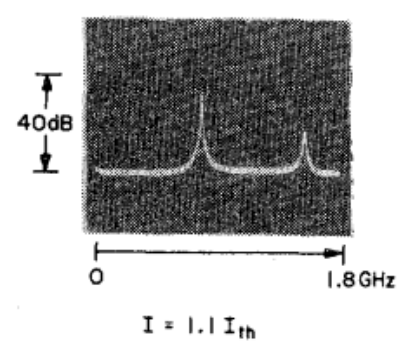

Fig. 5. Microwave spectrum of a self-pulsing laser.

a width of less than $10 \mathrm{MHz}$. The coupling results in approximately a 6-10 percent reduction in threshold. The induced resonance tracks the roundtrip frequency as the cavity length is varied, its amplitude exhibiting a maximum at some cavity length as shown in Fig. 4. At longer cavity lengths, the frequency locks onto the second external-cavity harmonic. Some of these observations have been seen by Broom [9] and Figueroa et al. [10]. This, in effect, is self-mode locking.

\section{B. Self-Pulsing Lasers}

We examined the effect of the external cavity on a selfpulsing lasers (including BH, proton stripe, and CSP lasers). The results described below are qualitatively similar in all the lasers examined. The microwave spectrum of the laser without an external cavity shows pulsations at about $800 \mathrm{MHz}$ (see Fig. 5 ) and this frequency increases with increasing current. When this laser is coupled to an external cavity less than approximately $5 \mathrm{~cm}$ long, the coupling does not seem to have much of an effect on the pulsing behavior. A small shift in the pulsing frequency is observed, but there is no correspondence with the external cavity roundtrip frequency. However, over the range of cavity lengths from about 6 to $11 \mathrm{~cm}$, the pulsation is significantly quenched [see Fig. 6(a)] and beyond $11 \mathrm{~cm}$ the pulsation amplitude grows with its frequency locked to the first cavity harmonic $(c / 2 L)$. The suppression of pulsations can be maintained only for bias currents below certain values. The coupling coefficient $\epsilon$ in this case is 1-5 percent. Suppression of self-pulsations can be accomplished even with a very small coupling coefficient. In the case shown in Fig. 6(b), the coupling coefficient is less than 1 percent, and the suppression is maintained for currents below 1.1 times threshold. A similar quenching effect has been observed with a piece of multimode fiber of optimum length as the external cavity [3]. Earlier experiments of Paoli et al. [7] with a much longer cavity 


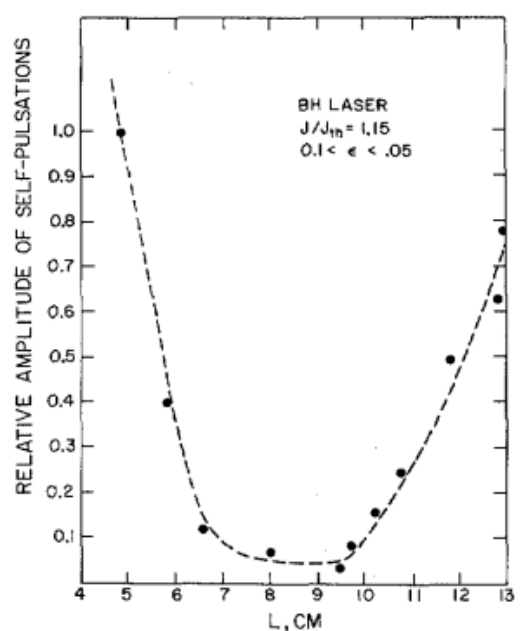

(a)

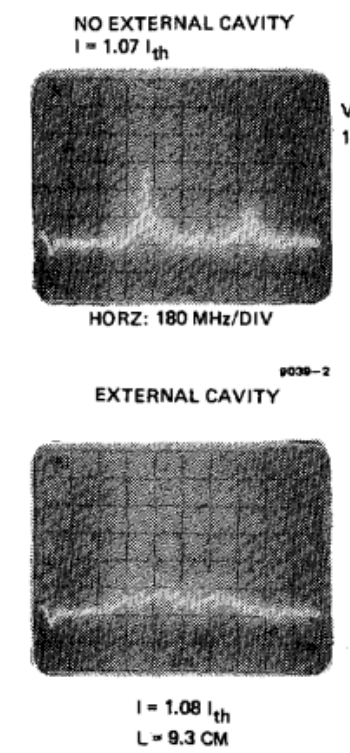

(b)

Fig. 6. (a) Amplitude of self-pulsing versus cavity; (b) microwave spectrum of a self-pulsing laser quenched by an external cavity 9.5 cm long.

length $(\simeq 75 \mathrm{~cm})$ did not show the quenching effect, and the pulsation frequency was observed to lock to an externalcavity harmonic other than the fundamental. As the cavity length was increased, they actually observed the pulsing frequency jumping from the $N$ th harmonic to the $N+1$ th harmonic at certain cavity lengths. It was also observed that when the external cavity feedback was applied with a roundtrip time near the laser's self-pulsing period, the optical pulse narrowed down to, in some cases, less than 160 ps. This, in essence, is passive mode locking. Addition of current modulation at the pulse repetition frequency would clearly enhance and shorten the pulse, as it is a combination of passive and active mode locking.

\section{Theoretical Calculations}

All the above experimental observations can be interpreted as consequences of a model of self-pulsation. Numerical calculations have been performed on Copeland's trap [11] model with external optical feedback. We specifically investigate the dependence of the pulsation characteristics on the external

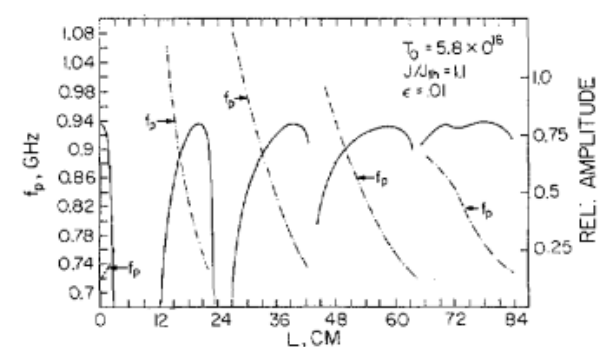

(a)

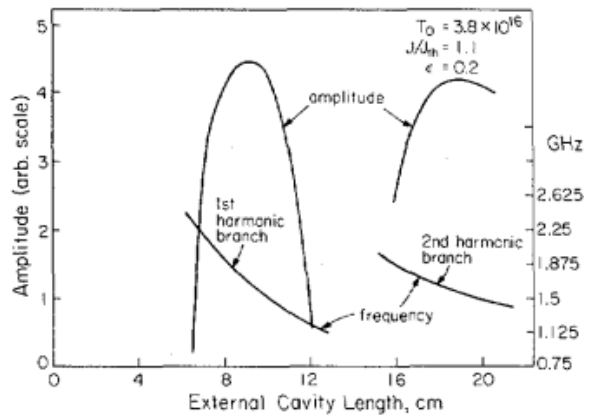

(b)

Fig. 7. Computed results of amplitude and frequency of sustained pulsation versus external cavity length: (a) for a self-pulsing laser; (b) for a nonpulsing laser. Spontaneous and photon lifetimes are, respectively, taken to be $3 \mathrm{~ns}, 2.9 \mathrm{ps}$ in (a) and $1.5 \mathrm{~ns}, 1.4 \mathrm{ps}$ in (b).

cavity length, and the results conform closely to that observed experimentally. This, however, is not a confirmation of the trap model, for other models including saturable absorber or superlinear gain yield similar results [12]-[19]. The equations and parameters used are the same as those in [3]. The use of the delayed photon feedback term [1], which neglect the optical phase, to describe the external cavity (which has been used by numerous other authors) can be justified as follows: for sharply pulsing solutions, the situation is that of one or more discrete photon packages bouncing back and forth between the laser diode and the external mirror. It is clear that in this case inclusion of the phase term is not necessary. In the case of nonpulsing (steady) or slowly varying solutions (slow compared to the external cavity roundtrip time), the result could be affected by coherence interference effects [20], [24]. However, if one neglects small variations (on the order of an optical wavelength) in the external cavity length, the overall picture can be well described by the above approach.

The computed results are shown in Fig. 7(a) and (b), which are plots of sustained pulsation amplitudes and frequencies versus external cavity length. Fig. 7(a) is calculated with $T_{0}$ above the critical pulsing value with a small coupling coefficient $(\epsilon=0.01)$, and it shows the quenching bands at short cavity lengths and frequency harmonic jumping at long cavity lengths, as observed experimentally. Fig. 7(b) is calculated with $T_{0}$ slightly below the critical value and with a large coupling coefficient $\epsilon=0.2$. It shows induced sustained pulsations at certain ranges of cavity length, and conforms qualitatively to our experimental plot, Fig. 4. For smaller trap densities or smaller coupling coefficients, it becomes increasingly difficult to induce sustained pulsations (i.e., a much larger $\epsilon$ is required) and even if it occurs, the pulses are far from being sharp. Notice that at very short cavity lengths, the pulsing character- 
istics of the laser are not altered: a self-pulsing laser continues to self-pulse, while a nonpulsing laser remains stable with the external cavity added. Therefore, the effects of pulse generation and quenching are not just due to a change in photon lifetime of the laser diode, they are retarded effects due to the external cavity.

\section{Analysis of Rate Equations WITH SATURABLE LOSS OR SUPERLINEAR GAIN-NYQUIST DIAGRAM APPROACH ${ }^{1}$}

As mentioned before, common to most proposed models for self-pulsations are two underlying effects; saturable loss and superlinear gain. It is thus reasonable to believe that a general saturable loss description is applicable to most lasers. The rate equations with a simple saturable absorbing loss of the form $L=L_{0} /\left(1+P / P_{s}\right)[22]$ are

$$
\begin{aligned}
& \dot{N}=J-N-N P \\
& \dot{P}=\gamma(N-1-L) P
\end{aligned}
$$

where $N$ is the electron density, $P$ is the photon density, $J$ is the pump current, and $\gamma$ is the ratio of the spontaneous to photon lifetime. Stability of the steady state is examined by small-signal analysis, where we write

$$
\begin{aligned}
& N=N_{0}+n \\
& P=P_{0}+p
\end{aligned}
$$

where $n$ and $p$ are small perturbations, and $N_{0}$ and $P_{0}$ are steady-state values.

Assuming solutions of the form $e^{s t}$, we have the following characteristic equation:

$$
f(s)=s+\gamma P_{0}\left(\frac{N_{0}}{s+\left(1+P_{0}\right)}-\alpha\right)=0
$$

where

$$
\alpha=\frac{L_{0}}{\left[1+\left(P_{0} / P_{s}\right)\right]^{2} P_{s}}
$$

As is well known, instability results if $s$ lies in the right half of the complex plane.

We shall perform stability analysis using Nyquist diagrams [21]. Although (2) is simple enough for direct analytic solution, the Nyquist diagram approach proves to be very useful in the case where external cavity feedback is introduced. The Nyquist plot is generated by mapping the contour $C$ on the complex plane into a new contour $\Gamma$ by an analytic function $f(z)$, where $C$ is the contour shown in Fig. 8. The number of times $\Gamma$ encircles the origin in the clockwise direction is the number of zeros minus the number of poles of $f(z)$ in the right half complex plane [21]. Fig. 9 shows a plot of $\Gamma$; the solid line is the locus $f(j \omega)$. We see that $\Gamma$ encircles the origin twice if

\footnotetext{
${ }^{1}$ After this manuscript had been submitted, Glasser published a paper in which a similar approach was used in stability analysis [25].
}

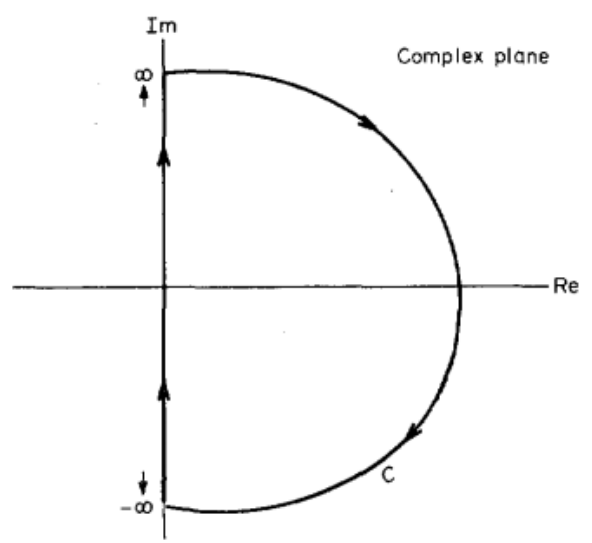

Fig. 8. The contour $C$ on the complex plane.

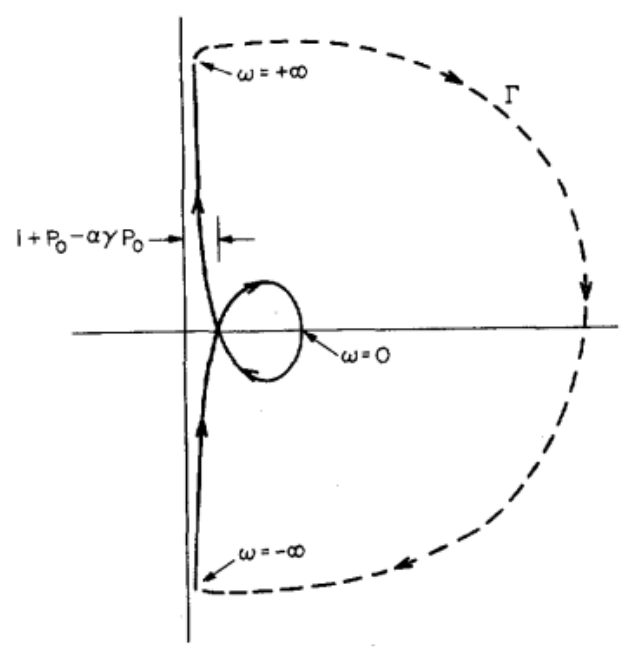

Fig. 9. Nyquist diagram for rate-equations including saturable absorbers.

$$
a>\frac{1+P_{0}}{\gamma P_{0}} .
$$

Under this condition, the zeros have positive real parts and pulsations start to build up. Expressed in terms of the absorber density $L_{0}$ and saturation photon density $P_{s}$ using (3), condition (4) can be written as

$$
\frac{L_{0}}{P_{s}} \geqslant \frac{1+P_{0}}{\gamma P_{0}}\left(1+\frac{P_{0}}{P_{s}}\right)^{2} \text {. }
$$

This result is similar to that of Haus [22] in analyzing parameter ranges for passively mode-locked lasers.

It is hardly surprising to find that a superlinear gain, a stimulated gain that takes the form $g=N P(1+\alpha P)$, where $N$ and $P$ are the electron and photon densities, respectively, would produce the same instability if $\alpha$ exceeds the amount given in (4).

\section{Characteristic Equation of the Combined LASER-ExTERnAL CAVITY SYSTEM}

The photon rate equation with a delayed feedback term is

$$
\dot{P}(t)=\gamma\left(N(t) P(t)-P(t)-\frac{L_{0} P(t)}{1+\left(P(t) / P_{s}\right)}+\epsilon P(t-\tau)\right)
$$

where $\tau$ is the roundtrip time of the external cavity, and $\epsilon$ is 
the coupling coefficient defined earlier. This $\epsilon$ can be estimated experimentally by the shift in lasing threshold. The steadystate solutions of the modified rate (6) is

$$
N_{0}=1+\frac{L_{0}}{1+\left(P_{0} / P_{s}\right)}-\epsilon .
$$

The small-signal photon equation is obtained in a straightforward manner:

$$
\dot{p}=\gamma\left[P_{0} n+\alpha P_{0} p+\epsilon(-p+p(t-\tau))\right]
$$

where $\alpha$ is defined as before [see (4)]. Note that as $\tau \rightarrow 0$, the feedback term disappears, showing that any quenching or induced pulsation effects are retarded effects not merely a change in photon lifetime.

Equation (8) is a difference-differential equation which does not lend itself to easy analytic solutions. However, its stability can be analyzed through Laplace transformation [23] and the use of Nyquist diagrams, simple expressions for minimum required $\epsilon$ and quenching bands can be obtained geometrically.

Laplace transforming equation (8) gives the following transcendental characteristic equation:

$$
\begin{aligned}
f_{f . b .}(s) & =s+\gamma\left[\frac{P_{0} N_{0}}{\left(1+P_{0}\right)+s}-\alpha P_{0}+\epsilon\left(1-e^{-s \tau}\right)\right]=0 \\
& =f(s)+\gamma \epsilon\left(1-e^{-s \tau}\right)
\end{aligned}
$$

where $f(s)$ corresponds to the laser without feedback.

\section{Quenching Range of Self-Pulsing Lasers Coupled to a Short External Cavity}

We first consider the case of a self-pulsing laser. The Nyquist diagram of a self-pulsing laser is shown in Fig. 10(a). The locus $f(j \omega)$ as parametrized by $\omega$ passes very close and to the left of the origin [as a consequence of the fact that the zeros of $f(s)$ lie very close to the imaginary axis]. Since the locus is symmetrical with respect to $\omega$, we will just look at the +ve branch of $\omega$. The portion of the locus closest to the origin approximates a vertical straight line at a distance $K=1+P_{0}$ $\alpha \gamma P_{0}$ to the left of the origin [see Fig. 10(b)]. The value of $\omega$ at that part of the locus is approximately equal to $\omega_{0}$, the imaginary part of the zero of $f(s)$, which corresponds roughly to the pulsing frequency of the laser. (The actual frequency is somewhat lower due to the large-signal effect.) When feedback is included, the locus becomes $f_{f . b .}(j \omega)=$ $f(j \omega)+\gamma \epsilon\left(1-e^{-j \omega \tau}\right)$. The effect of this additional term on the portion of the locus closest to the origin is shown in Fig. 11: the locus is shifted to the right by an amount $\gamma_{\epsilon}(1-$ $\left.\cos \omega_{0} \tau\right)$. For sufficiently large $\epsilon$ and a suitable delay $\tau$, we see that the locus would no longer encircle the origin-the system becomes stable and pulsations are quenched.

From Fig. 11 we can see that the minimum amount of coupling $\epsilon$ required is

$$
\epsilon_{\min }=-K / 2 \gamma
$$

where $K=1+P_{0}-\gamma P_{0} \alpha<0$ for a self-pulsing laser, $P_{0} \simeq$ $J^{\prime}-1, J^{\prime}$ is the pump current, and $\alpha$ is as defined previously [see (3)]. The range of external cavity roundtrip time $\tau$ for which quenching occurs is

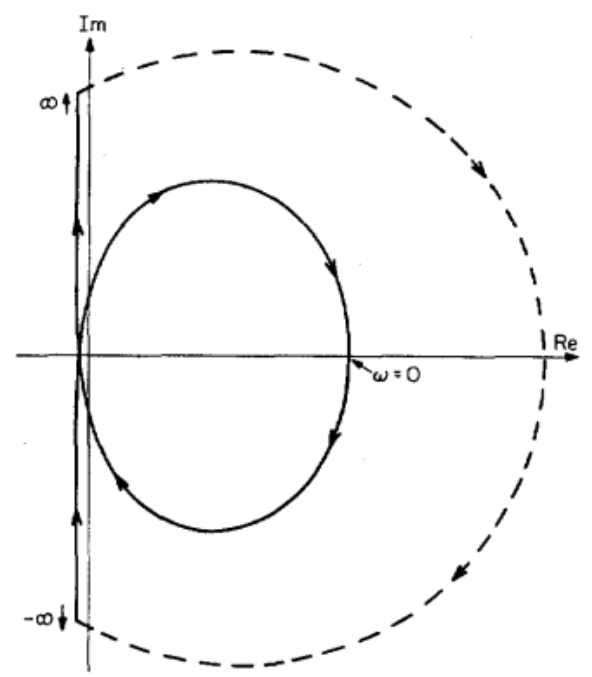

(a)

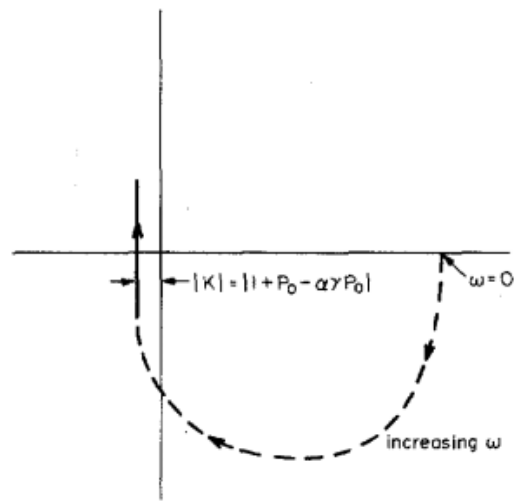

(b)

Fig. 10. (a) Nyquist diagram for a self-pulsing laser and (b) approximate locus near the origin. Only the $+v e_{i} \omega$ branch is shown.

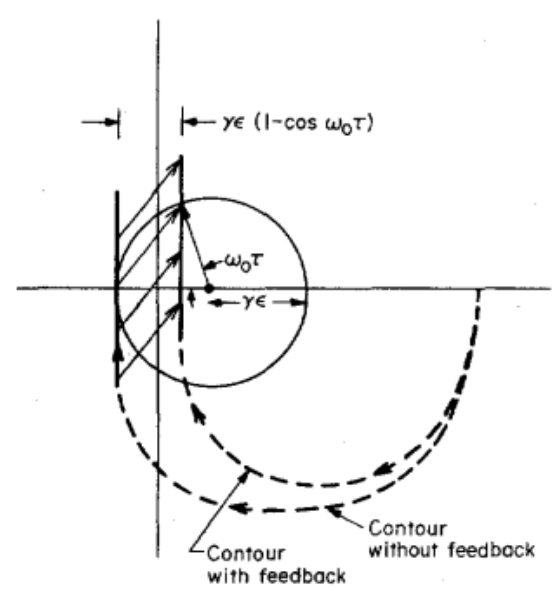

Fig. 11. Nyquist diagram illustrating the effect of external cavity on a self-pulsing laser.

$$
\frac{1}{\omega_{0}}\left(2 \pi-\cos ^{-1} \phi\right)>\tau>\frac{1}{\omega_{0}} \cos ^{-1} \phi
$$

where $\phi=(1-K / \gamma \epsilon)$ and $\omega_{0}$ is approximately the self-pulsation frequency of the laser. We note that a very small $\epsilon$ is sufficient for quenching. Take a value of $L_{0}=0.02$ (which gives self-pulsation frequencies that fit typical experimental data well). If we assume that the laser is operated at 1.1 threshold, $\gamma=1000$, we have $\epsilon_{\min } \simeq 0.002$. Of course, this amount of $\epsilon$ 


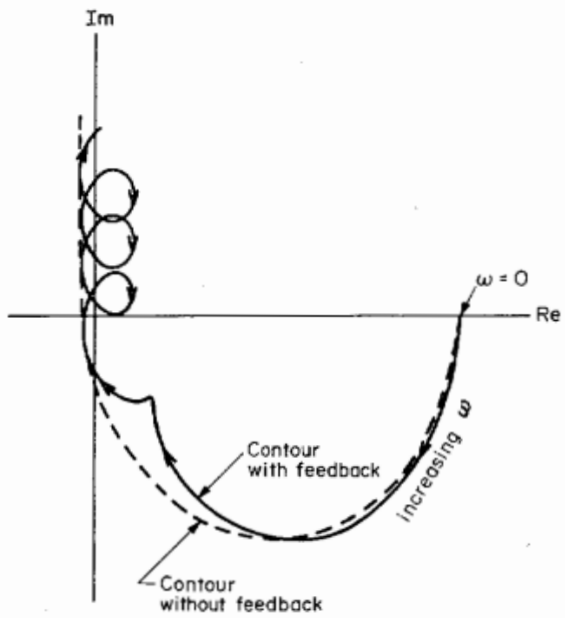

Fig. 12. Nyquist diagram for a self-pulsing laser coupled to a long cavity.

just barely pushes the zeros of the characteristic equation across the imaginary axis into the left half plane. To have a significant quenching effect, a larger $\epsilon$ is required so that the zeros lie deeper into the left half plane, which leads to a large damping.

To estimate the quenching band, we use the above numerical values, resulting in a self-pulsation frequency $\omega_{0}$ of about 0.8 GHz. Then $K=1+P_{0}-\gamma \alpha P_{0} \simeq-0.49$, let $\epsilon=0.01$, and the quenching band is calculated using (11) to lie approximately between 3 and $15 \mathrm{~cm}$. This is compared with the numerical results shown in Fig. 7(a) calculated using the trap model. The trap density used in this case produces self-pulsation at roughly the same frequency as above $(0.8 \mathrm{GHz})$ and the same coupling coefficient $\epsilon=0.01$ is used. The approximate agreement shows that the above analysis of the quenching effect is quite general and model independent.

Thus, it appears that as the external cavity length is increased ( $\tau$ increased), we would see alternating bands of quenching and pulsations. However, the above analysis is suitable only for short cavity lengths (which is where the quenching bands occur). For long cavity lengths (large $\tau$ ), the Nyquist plot takes the form of a helix (viewed in two dimensions) as shown in Fig. 12. The origin could not escape the fate of being enclosed, and thus quenching effects do not occur at long cavity lengths, as shown in the pulsation amplitude versus cavity length plot of Fig. 7(a).

It is also obvious from (10) that, at least for current ranges not too much higher above threshold $(\gtrsim 1.7$ times threshold in our numerical example, which we usually do not encounter in practice), it becomes increasingly difficult to quench the pulsations at higher bias current-which is what we observed in our experiments.

\section{NONPUlsing LASERS COUPled to EXTERNAL Cavities-The Microwave Gain Lineshape AND Mode Structure of THE COMBINED SYSTEM}

Nonpulsing lasers, when coupled to external cavities, can be made to self-pulse with a pulsewidth sometimes as short as 5 ps [8]. As mentioned in Section II, such induced pulsing occurs only over a certain range of the external cavity lengths.
In some cases, induced self-pulsing occurs at twice the external cavity roundtrip frequency. It is also a common experience, when working with lasers coupled to long fiber pigtails, to observe on the microwave spectrum of the optical output a cluster of spikes around 1-2 GHz, and the frequency separation between spikes corresponds to the inverse of the pigtail roundtrip time. All of the above mentioned hitherto complex pulsating phenomena can be explained very intuitively by interpreting the combined laser-external cavity system as a microwave oscillator with a limited gain band and discrete mode structures. The small-signal microwave gain lineshape and the mode structures will be derived analytically.

As mentioned before, the dynamic behavior of the system is determined by the zeros of $f_{f . b}$. $(s)$ [see (9)]. Due to the transcendental term, $f_{f . b}$. $(s)$ has an infinite number of complex zeros $g_{k} \pm i \omega_{k}, k=1,2, \cdots$. Alternatively, we can define a continuous function $g(\omega)$ such that $g\left(\omega_{k}\right)=g_{k}$. The imaginary part of the zeros $\omega_{k}$ indicates a resonant peak at frequency $\omega_{k} / 2 \pi$ and these are the modes of the system. The system will spontaneously oscillate at frequency $\omega_{k}$ if $g\left(\omega_{k}\right)>0$, i.e., the response grows as $\exp \left(g_{k} t\right) \exp \left(i \omega_{k} t\right) . g(\omega)$, as a function of $\omega$, can thus be interpreted as the microwave gain curve of the system. The system is "above threshold" if $g(\omega)>$ 0 over a certain range of $\omega$.

The gain curve $g(\omega)$ can be derived with the help of the Nyquist diagram. This was done in the Appendix, resulting, for frequencies near the peak of the gain curve, in

$$
g(\omega)=-\frac{1}{2 \gamma \epsilon \omega^{2}}\left[\begin{array}{l}
\omega^{4}-2 \gamma P_{0}\left(N_{0}+\gamma \epsilon \alpha\right) \omega^{2} \\
+\gamma^{2} P_{0} N_{0}\left(P_{0} N_{0}+2 \epsilon\left(1+P_{0}\right)\right)
\end{array}\right]
$$

and the mode frequencies

$$
\omega_{k}=(2 \pi k-\theta) / \tau
$$

where $\tau$ is the external cavity roundtrip time, and $\theta$ is a small frequency pulling term given by

$$
\theta \simeq \frac{1}{\gamma \epsilon} \operatorname{Im}(f(2 \pi k i)) \text {. }
$$

Fig. 13 shows a plot of $g(\omega)$ for various coupling coefficients $\epsilon$, with $\gamma=1000, P_{0}=0.3, N_{0}=1, \alpha=3.9 \times 10^{-3}, \tau_{s}$ (= spontaneous lifetime $)=3$ ns. As expected, the system is "below threshold" unless $\epsilon$ is above a certain $\epsilon_{\min }$, and the linewidth widens with further increase in $\epsilon$. For the modes near the line center (peak) of $g(\omega)$, the frequency pulling term $\theta$ is approximately given by $\theta=\operatorname{Im}\left[f\left(\omega_{0}\right)\right] / \gamma \epsilon$, where $\omega_{0}$ is the line center frequency. For the above parameters, $\theta$ is approximately $0.05 \mathrm{rad}$ so that the modes are virtually that of the passive external resonator.

We thus have the condition for sustained microwave pulsation; first, the coupling between the laser and the external cavity must be above a critical value so that there is a certain frequency range over which $g(\omega)$ is $>0$. Then, at least one or more modes given by (13) must lie within that frequency range. This range can be easily found by setting $g(\omega)=0$, giving

$$
\omega_{ \pm}^{2}=\gamma P_{0}\left(N_{0}+\gamma \epsilon \alpha\right) \pm \gamma^{2} P_{0} \alpha \sqrt{\epsilon\left(\epsilon-\epsilon_{\min }\right)}
$$

where 


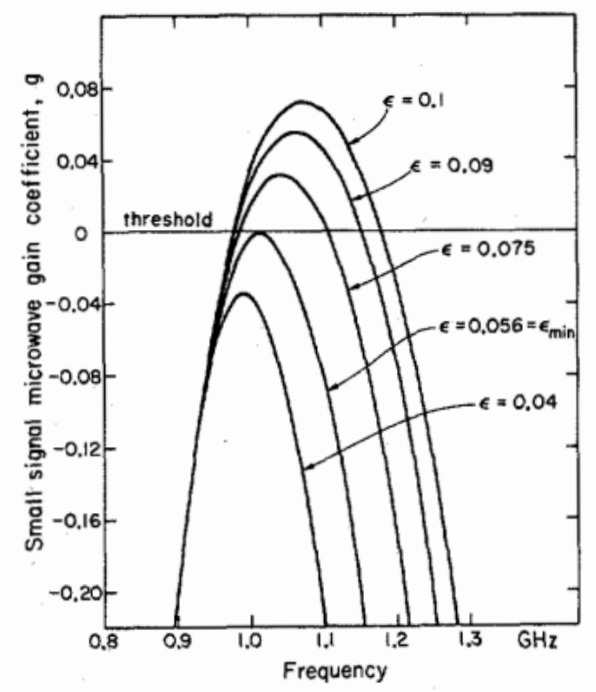

Fig. 13. The small-signal microwave gain lineshape of the combined laser-external cavity system for various coupling coefficients $\epsilon$.

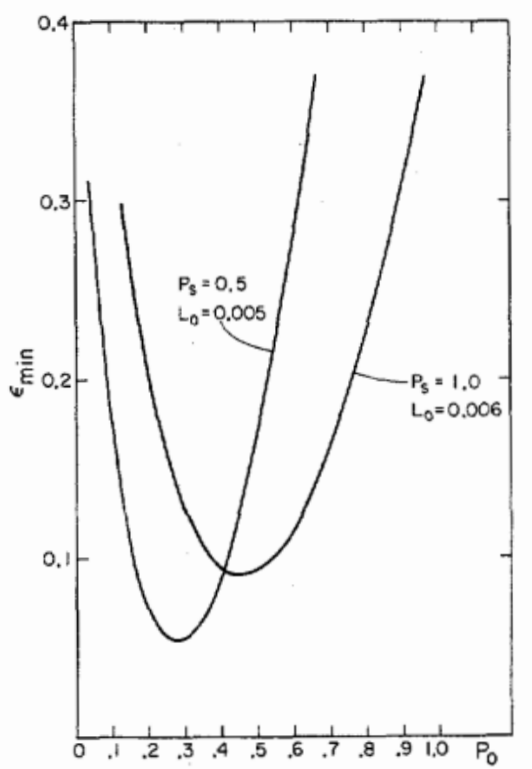

Fig. 14. Plot of $\epsilon_{\min }$ required for induced pulsation in nonpulsing lasers.

$$
\epsilon_{\min }=2 N_{0} K / \alpha^{2} \gamma^{2} P_{0}
$$

is the minimum coupling for induced pulsing. The quantity $K$ defined as $K=1+P_{0}-\gamma P_{0} \alpha$ is an indication of how close the laser is to self-pulsing, and in the case when it is negative, it measures how deep the laser is into self-pulsing. The solid lines in Fig. 14 are plots of $\epsilon_{\min }$ versus the bias level as measured by the static photon density $P_{0}$ for two cases: 1)absorber density $L_{0}=0.005$ and saturation photon density $P_{s}=0.5$; 2) $P_{s}=1$ and $L_{0}=0.006(\gamma$ is taken to be 1000). [The calculated minimum absorber densities $L_{0}$ for self-pulsing are $L_{0} \simeq 0.006$ and 0.007 , respectively, for cases 1 ) and 2).] We observe from Fig. 14 that a very high coupling coefficient is required for inducing pulsation if the bias level is not optimized.

The hitherto complex behavior of the laser diode coupled to external cavities of various lengths can now be understood in very intuitive terms. When the external cavity length is very short, the microwave mode frequencies are very high and do not fall under the positive gain line. In fact, the value of $g(\omega)$

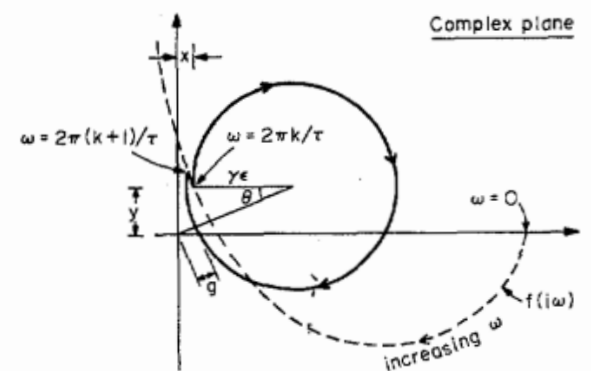

Fig. 15. Nyquist diagram for the derivation of $g(\omega)$ and the mode spectrum.

is large and negative at high frequencies so that a short cavity actually serves to stabilize the laser diode. As the EC length is increased, the first microwave mode $(k c / 2 L, k=1)$ falls within the gain line and induced pulsation occurs at the fundamental roundtrip frequency of the EC. Further increase in the EC length brings the $k=1$ mode outside the gain line, pulsation ceases until the $k=2$ mode moves in and induced pulsations occur at twice the EC roundtrip frequency, and so on. For very long $\mathrm{EC}$ 's, several microwave modes lie within the gain line simultaneously, and "multimode" microwave oscillation occurs, as often observed in lasers coupled to very long fiber pigtails. Badly degrading lasers, with a large absorbing defect density, are particularly vulnerable to such disturbance, as indicated by (16).

\section{CONCLuSION}

The behavior of various laser diodes operating in external cavities can thus be related to the strength of saturable absorbers in the lasers. The ability of the laser to generate short pulses is correlated with the amount of saturable absorber present in the laser medium. Although the above analysis does not reveal the nature of the absorbers, it illustrates the various effects the external cavity has on the laser. Thus, useful devices can be fabricated by introducing known amounts of absorbers into the laser, and the generated pulses can be sharpened and frequency stabilized by an external cavity. On the other hand, self-pulsing lasers can be made useful by quenching it in an external cavity of appropriate length. The use of optical fibers as external cavities can render actual systems highly compact and stable, which is desirable in any practical application.

\section{APPENDIX}

We shall derive $g(\omega)$ and the mode frequencies $\omega_{k}$ 's with the help of the Nyquist diagram-a plot of $f_{f . b .}$. $(i \omega)$ in the complex plane. We assume that one or more zeros of $f_{f . b} .(s)$ lie very close to the imaginary axis. Then, the curve $f_{f . b}(i \omega)$ makes its closest approach to the origin when $\omega$ equals the imaginary part $\omega_{k}$ of a zero, and the distance of closest approach is the real part $g_{k}$ of that zero. $g_{k}$ is by convention positive(negative) if the locus does(does not) encircle the origin.

To see what $f_{f . b}(j \omega)$ looks like, we first plot $f(j \omega)$ (the term without feedback) as shown by the dashed line in Fig. 15. This curve will itself encircle the origin if $K>0$, as de-

${ }^{2}$ Since $f(-j \omega)=f^{*}(j \omega)$, the locus is symmetric about the real axis so that only the $+v e \omega$ branch is considered. 
fined in (10), i.e., the laser self-pulses. Now we divide this curve into tiny segments with end points parametrized by $\omega=$ $2 \pi k / \tau$ and $\omega=2 \pi(k+1) / \tau, k=0,1,2, \cdots$. Then, it is obvious that addition of the feedback term $\gamma \epsilon(1-\exp (-i \omega \tau))$ to $f(j \omega)$ transforms each segment into (roughly) a circle of radius $\gamma \epsilon$, as shown in Fig. 15. From simple trigonometry, the closest approach distance of this circle to the origin is $g=\gamma \epsilon-$ $\sqrt{(\gamma \epsilon+x)^{2}+y^{2}}$, where $x$ and $y$ are, respectively, the real and imaginary part of $f(j \omega)$ :

$$
\begin{aligned}
& x(\omega)=\frac{\gamma\left(1+P_{0}\right) P_{0} N_{0}}{\left(1+P_{0}\right)^{2}+\omega^{2}}-\gamma P_{0} \alpha \\
& y(\omega)=\omega\left(1-\frac{\gamma P_{0} N_{0}}{\omega^{2}+\left(1+P_{0}\right)^{2}}\right) .
\end{aligned}
$$

This value $g$ is then the real part of a zero of $f_{f . b .}(s)$; the imaginary part $\omega_{k}$ of this zero is the value of $\omega$ at which the closest approach occurs. From Fig. 15, we have

$$
\omega_{k} \tau=2 \pi k-\theta
$$

where $\theta$ is the angle as shown,

$$
\theta \simeq \tan ^{-1}\left[\frac{y(2 \pi k i)}{\gamma \epsilon+x(2 \pi k i)}\right] \text {. }
$$

In the frequency range of interest (where the locus is closest to the origin), $\omega \gg 1+P_{0}$ and for induced pulsation to occur $\epsilon$ has to be sufficiently large such that $\gamma \epsilon \gg x, y$. Making these approximations and substituting (A1) into $g$, we obtain the gain spectrum (12), and the mode frequencies (13) and (14).

\section{REFERENCES}

[1] R. P. Salathe, "Diode lasers coupled to external resonators," Appl. Phys., vol. 20, p. 1, 1979.

[2] N. Chinone, K. Aiki, and R. Ito, "Stabilization of semiconductor laser outputs by a mirror close to a laser facet," Appl. Phys. Lett., vol. 33, p. 990, 1978.

[3] L. Figueroa, K. Y. Lau, and A. Yariv, "Intensity self-pulsations in ( $\mathrm{GaAl})$ As injection lasers operating in an external cavity," Appl. Phys. Lett., vol. 36, p. 248, 1980.

[4] P. T. Ho, L. A. Glasser, E. P. Ippen, and H. A. Haus, "Picosecond pulse generation with a CW (GaAl)As diode," Appl. Phys. Lett., vol. 33, p. 241, 1978.

[5] P. T. Ho, "Coherent pulse generation with GaAlAs laser by active mode-locking," Electron. Lett., vol. 15, p. 526, 1979.

[6] H. Kuwahara, "Simulation on intensity self-pulsation in CW semiconductor lasers," Appl. Phys., vol. 20, p. 67, 1979.

[7] T. L. Paoli and J. E. Ripper, "Frequency stabilization and narrowing of optical pulses from CW GaAs injection lasers," IEEE $J$. Quantum Electron., vol. QE-6, pp. 335-339, June 1970.

[8] E. P. Ippen, D. J. Eilenberger, and R. W. Dixon, "Picosecond pulse generation by passive modelocking of diode lasers," to be published.

[9] R. F. Broom, E. Mohn, C. Risch, and R. Salatha, "Microwave self-modulation of a diode laser coupled to an external cavity," IEEE J. Quantum Electron., vol. QE-6, pp. 335-338, June 1970.

[10] L. Figueroa, C. Slayman, and H. W. Yen, "Optical-microwave interaction in semiconductor devices," Hughes Res. Lab. Quarterly Rep., Contract N00173-78-C-0192, Apr. 1979.

[11] J. A. Copeland, "Semiconductor-laser self-pulsing due to deep level traps," Electron. Lett., vol. 14, p. 809, 1979.

[12] N. G. Basov, "Dynamics of injection lasers," IEEE J. Quantum Electron., vol. QE-4, pp. 855-867, Nov. 1968.

[13] T. P. Lee and R. H. R. Roldan, "Repetitive $Q$-switched light pulses from GaAs injection lasers with tandem double-section strip geometry," IEEE J. Quantum Electron., QE-6, pp. 339-352, June 1970.
[14] T. L. Paoli and J. E. Ripper, "Coupled longitudinal mode pulsing in semiconductor lasers," Phys. Rev. Lett., vol. 22, p. 1085, 1969.

[15] D. Kato, "Microscale degradation in (GaAl)As double-heterostructure diode lasers," Appl. Phys. Lett., vol. 31, p. 588, 1977.

[16] R. W. Dixon and W. B. Joyce, "A possible model for sustained oscillations (pulsations) in (AlGa)As double-heterostructure lasers," IEEE J. Quantum Electron., vol. QE-15, pp. 470-474, June 1979.

[17] J. P. Van der Ziel, J. L. Merz, and T. L. Paoli, "Study of intensity pulsations in proton-bombarded strip-geometry double-heterstructure $\mathrm{Al}_{\mathrm{x}} \mathrm{Ga}_{1-\mathrm{x}}$ As lasers," J. Appl. Phys., vol. 7, p. 4620, 1979.

[18] B. W. Hakki, "Instabilities in output of injection lasers," J. Appl. Phys., vol. 50, 1979.

[19] R. L. Hartman, R. A. Logan, L. A. Koszi, and W. T. Tsang, "Pulsations and absorbing defects in (AlGa)As injection lasers," J. Appl. Phys., vol. 50, p. 4616, 1979.

[20] T. Kanada and K. Nawata, "Injection laser characteristics due to reflected optical power,"IEEE J. Quantum Electron., vol. QE-15, pp. 559-565, July 1979.

[21] H. W. Bode, Network Analysis and Feedback Amplifier Design. New York: Van Nostrand, 1959.

[22] H. A. Haus, "Parameter ranges for CW passive mode-locking," IEEE J. Quantum Electron., QE-12, p. 169, 1976.

[23] N. Minorsky, Nonlinear Oscillations. Krieger, 1974.

[24] R. Lang and K. Kobayashi, "External optical feedback effects on semiconductor injection laser properties," IEEE J. Quantum Electron., vol. QE-16, pp. 347-355, Mar. 1980.

[25] L. A. Glasser, "A linearized theory for the diode laser in an external cavity," IEEE J. Quantum Electron., vol. QE-16, pp. 525531, May 1980.

Kam Y. Lau, photograph and biography not available at the time of publication.

Luis Figueroa, photograph and biography not available at the time of publication.

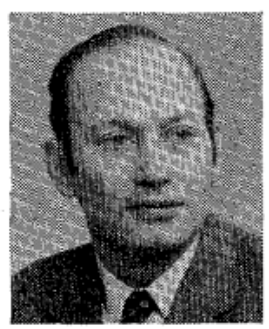

Amnon Yariv (S'56-M'59-F'70) was born in Tel Aviv, Israel, on April 13, 1930. He received the B.S., M.S., and Ph.D. degrees in electrical engineering from the University of California, Berkeley, in 1954,1956, and 1958, respectively. He was a veteran of the Israeli war of independence. In 1959 he joined Bell Laboratories, Murray Hill, NJ, where his research included the early stages of the laser effort. In 1964 he joined the California Institute of Technology, Pasadena, CA, as an Associate Professor of Electrical Engineering, becoming a Professor in 1966. On the technical side, he took part (with various co-workers) in the discovery of a number of early solid-state laser systems, in the formulation of the theory of parametric quantum noise, and the prediction of parametric fluorescence, in the invention of the technique of mode-locked ultrashortpulse lasers and FM lasers, in the introduction of GaAs and CdTe as infrared electrooptic and window materials, and in proposing and demonstrating semiconductor lasers integrated optics technology. His present research efforts are in the areas of nonlinear optics, free electron lasers, semiconductor lasers, and integrated optics. He authored or coauthored 150 papers in professional journals as well as two books: Quantum Electronics (Wiley, 1967, 1975) and Introduction to Optical Electronics (Holt, 1971, 1976). He was an Associate Editor for the IEEE Journal of Quantum Electronics and is an Associate Editor of Optics Communications and the Journal of Applied Physics.

Dr. Yariv is a member of the American Physical Society, Phi Beta Kappa, and the National Academy of Engineering, and a Fellow of the Optical Society of America. 